\title{
AUREOMICINA ORAL EN LA MENINGITIS AGUDA PURULENTA DEL NIÑO
}

\author{
Prof. Julio MENeghello y Dra. CaRmen aguilo
}

Cátedra de Pediatría de los Profs. A. Baeza Goñi y J. Meneghello, - Hospital Manuel Arriarán

Desde hace algunos años nos hemos preocupado del tratamiento de la meningitis purulenta, usando los antibióticos (penicilina y estreptomicina) asociados generalmente a los sulfamidados. Los resultados obtenidos con esta terapéutica fueron relativamente satisfactorios, ya que se logró bajar considerablemente la mortalidad de esta grave afección $(1,7)$. Sin embargo, el número de enfermos que quedan con secuelas neuropsíquicas justifican plenamente el ensayo de nuevos antibióticos que permitan la curación en un. mayor número de enfermos y con el menor daño posiblf

Consideramos que la aureomicina podía ser de utilidad en el tratamiento de la meningitis purulenta, porque 1) los gérmenes que la producen con mayor frecuencia, como el Diplococcus pneumoniae y el Haemophylus influenzae, son sensibles a dicho antibiótico $(10)$; 2) las experiencias de Whitlock y col. (10) demuestran el pasaje de la droga al LCR. después de su administración oral, y el uso de una sola droga por vía oral significaba, además, la simplificación del tratamiento. Por otra parte, ya ha sido señalada en la experiencia extranjera $(2,3$. $4,6,9$, que es posible obtener en clínica el control de la meningitis purulenta con la aureomicina.

Material y pauta terapéutica. Nuestro material está constituído por aquellos enfermos con meningitis purulenta que ingresaron al Servicio de Infecciosos de la Sección A de -Medicina del Hospital M. Arriarán, durante los meses comprendidos entre julio y noviembre de 1952. Durante este tiempo un solo enfermo no recibió aureomicina, por haberse administrado otro antibiótico por más de 24 horas durante su hospitalización.

Se trataron 16 pacientes, cuya edad fluctuó entre 1 y 20 meses, con excepción de una niña de 7 años. La etiología de la infección meningea fué variada, en 6 casos el agente etiológico fué el D. pneumoniae; en 2 , el $\mathrm{H}$. influenzae; en 1, ambos gérmenes; en 1 , la $N$, meningitidis $y$ en 6 no se encontró el agente causal. El foco de origen probable fué en 5 niños una infección ótica aguda; en 1, empiema pleural; en 1, neumonía, y en los 9 casos restantes no pudo ser precisado. En el cuadro 1 se encuentran detallados los caracteres generales de nuestros enfermos.

La droga se indicó en todos los enfermos exclusivamente por vía oral, con excepción de una enferma (caso 11), que recibió una inyección endovenosa de $100 \mathrm{mgrs}$. Solamente en los dos primeros casos de nuestra serie se asoció sulfadiazina $(0,40 \mathrm{gr}$. por kgr. de peso en 24 horas). La dosis diaria de aureomicina fué de más o menos 100 mgrs. por kgr. de peso en 24 horas, fraccionada en 4 dosis parciales (cada 6 horas). Se administró en forma de cápsulas enteras o diluidas en leche. El tratamiento se prolongó por un lapso fluctuante entre 9 y 23 djas.

Resultados y comentarios. Teniendo presente que nuestra casuística es reducida, no es posible valorar los resultados del tratamiento en relación con las diversas caracteristicas de los enfermos (edad, etiología, gravedad de la infección). Hemos agrupado nuestros pacientes de acuerdo con la distinta evolución observada en: Grupo I, la infección. meníngea curó con la aureomicina exclusivamente y Grupo II, la infección necesitó de otro antibiótico.

En el grupo de pacientes (cuadro 2) que mejoraron exclusivamente con aureomicina, la regresión de los síntomas fundamentales de la enfermedad ocurrió en plazos similares a los observados en nuestras experiencias anteriores (1-7). Así, la fiebre desapareció generalmente en el curso de la primera semana; sólo en 2 pacientes no se normali- 
CUADRO N: $\mathrm{i}$

Algunas características generales đe 16 enfermos con meningitio purulenta tratados con aureomicina orat

\begin{tabular}{|c|c|c|c|}
\hline N." & Edad & Etiologia de la meningitis & Probable foco de origen \\
\hline $\begin{array}{r}1 \\
2 \\
3 \\
4 \\
5 \\
6 \\
7 \\
8 \\
9 \\
10 \\
11 \\
12 \\
13 \\
14 \\
15 \\
16\end{array}$ & $\begin{array}{l}8 \mathrm{~m} . \\
18 \mathrm{~m} . \\
11 \mathrm{~m} . \\
4 \mathrm{~m} . \\
2 \mathrm{~m} . \\
6 \mathrm{~m} . \\
4 \mathrm{~m} . \\
8 \mathrm{~m} . \\
20 \mathrm{~m} . \\
3 \mathrm{~m} . \\
7 \mathrm{a} . \\
2 \mathrm{~m} . \\
11 \mathrm{~m} . \\
1 \mathrm{~m} . \\
5 \mathrm{~m} . \\
5 \mathrm{~m} .\end{array}$ & $\begin{array}{c}\text { D. pneumoniae } \\
\text { D. pneumoniae } \\
\text { D. pneumoniae } \\
\text { D. pneumoniae } \\
\text { D. pneumoniae } \\
\text { D. pneumoniae } \\
\text { D. pneumoniae y H. influenzae } \\
\text { H. influenzae } \\
\text { H. influenzae } \\
\text { N. meningitidis } \\
\text { Sin germen } \\
\text { Sin germen } \\
\text { Sin germen } \\
\text { Sin germen } \\
\text { Sin germen } \\
\text { Sin fermen } \\
\end{array}$ & $\begin{array}{c}\text { Otitis izquierda } \\
\text { Otitis bilateral } \\
\text { Empiema pleural derecho } \\
= \\
\text { Neumonia izquierda } \\
\text { Otitis bilateral } \\
\text { Otitis derecha } \\
= \\
\text { Otitis derecha }\end{array}$ \\
\hline
\end{tabular}

GI!ADRO N. ${ }^{\circ} 2$

Algunos caracteres de los enfermos de meningitis aguda purulenta tratados con éxito con aureomicina

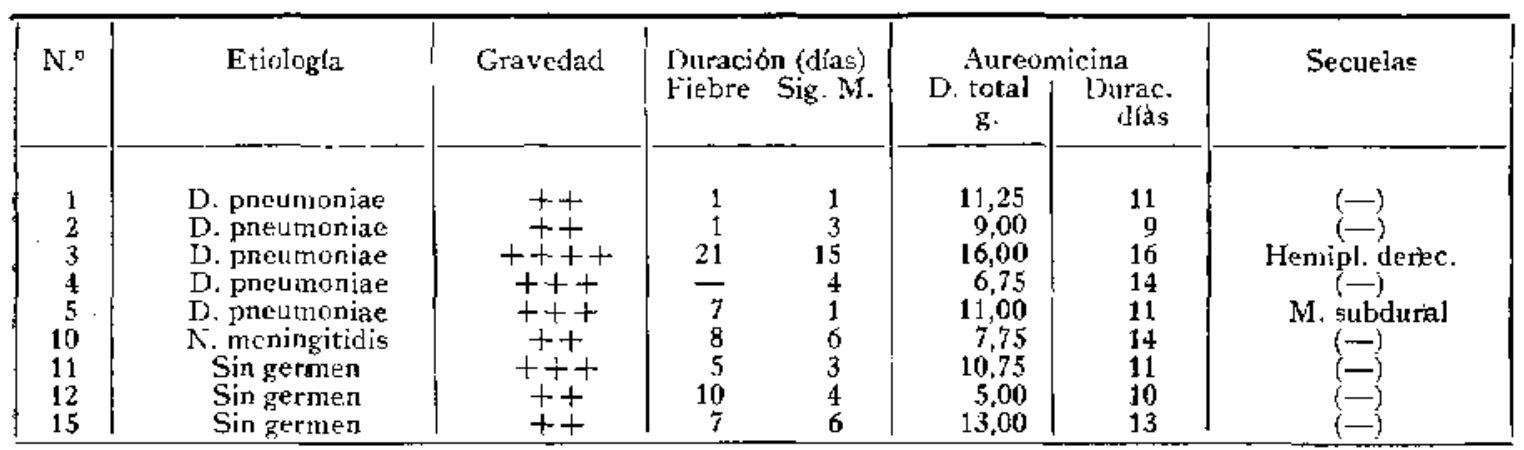

zć la ternperatura en este periodo (casos 3 y 12). E1 caso 3 se refiere a un lactante que ingresó, al Servicio de Infecciosos, prácticamente agónico con una hemiplegia plenariente establecida y en él la persistencia de la fiebre por 21 días puede atribuirse más bien a la lesión encefálica que al proceso infeccioso ya que el LCR, al $14^{9}$ día de hospitalización, tenía la ghucosa normal, una Iigera albuminorraquia y 48 elementos celulares. En cuanto al caso 12 cuya fiebre duró 10 días, debe quedar establecido que presentó sólo febrículas, no superiores a $37,5^{\circ} \mathrm{C} \mathrm{y}$ que la mejoría clínica y del LCR, se produjo en forma mucho más rápida. La evolución dc los signos meníngeos varió también en plazos semejantes. Igualmente en este lapso se apreció regresión evidente de las alteraciones del LCR (desaparición de los glóbulos de pus, normalización de ląs cifras de glucosa, franca disminución de la albuminosis). Esta es la impresión general del tratamiento en este grupo de enfermos pero hubo casos que mejoraron en forma rapidísima en tanto que otros lo hicieron más arrastradamente. Un ejemplo de ambos tipos de evolución lo tenemos en las observaciones de los casos 2 y 10.

Caso 2. (gráfico 1). Niño de 18 meses de edad, en deficientes condiciones nutritivas. (Peso de 6.800 grs.) que permaneció hospitalizado en el Servicio de Urgencia durante 5 días por un cuadro de carácter broncopulmonar y que fué tratado con penicilina procaína. Durante esos días el niñ̃o permaneció febril, aumentó el compromiso del estado general $\mathbf{y}$ aparecieron finalmente algunos signos meningeos. Se sospechó entonces que se trataba de una meningitis aguda que fué comprobada por la P. L. que dió salida a líquido 
purulento cuyo cultivo reveló la presencia de un neumococo. Se inició el tratamiento con aureomicina oral y se observó que la fiebre, atribuíble a la meningitis, desapareció en 48 horas, los signos meningeos, en 5 días y ei LCR, al $7^{\circ}$ día, estaba prácticamente normal. La fiebre que presentó después de las 48 horas correspondió a una infección gripal que no comprometió en absoluto el estado general. Curó sin secuelas neuropsíquicas aparentes en la fecha del alta.

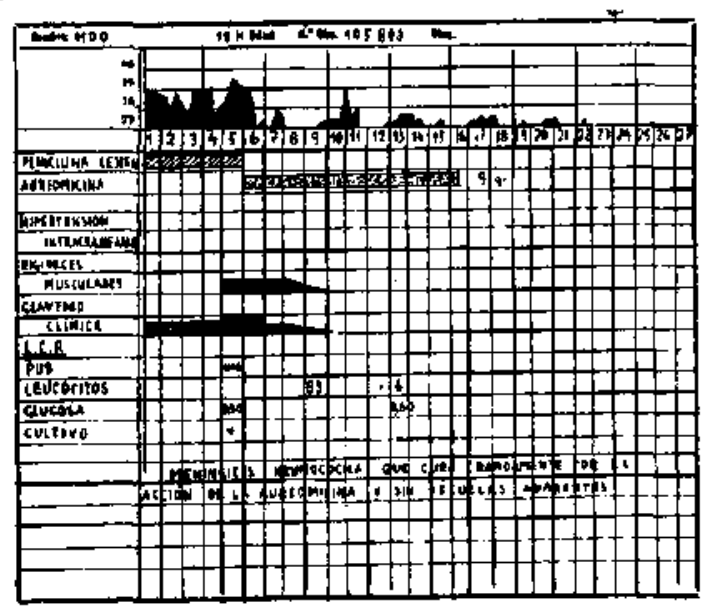

Gráfico 1

Mario D. M., I8 meses, Meningitis neumocócica que se inició $\mathrm{g}$ días antes de su ingreso con fiebre, in. tranquilidad, insomnio, decaimiento $y$ finalmente crisis convulsiva. A su ingreso se comprobó niño de. caddo, febril, con intensa disnea y ausencia de sig. nos meníngeos. Se trata con penicilina lenta. Al jo día se comprueba cierto grado de obnubilación sen. sorial y rigidez dolorosiz de la nuca. La temperatura continuaba alta. La $P$. L, confirma e] diagnóstico de meningitis. Se trata con aureomicina y cura rapidamente sin secuelas aparentes.

Fotografía al alta: nin̂o con actividad normāl.

Caso 10. (gráfico 2). En el gráfico correspondiente puede apreciarse la evolución seguida por un lactante de 3 meses de edad que sufre una meningitis meningocócica de mediana gravedad cuya sintomatología se había iniciado el día anterior a su ingreso. A pesar de ello, la evolución se hizo en forma arrastrada evidenciable especialmente en la duración del cuadro febril y alteraciones del LCR. Desarrolló un derrame subdural que se agotó rápidamente con las punciones evacuadoras y curó luego sin secuelas aparentes.

En este Grupo I de enfermos, se produjo derrame subdural, durante la evolución del cuadro meníngeo o en su convalescencia, en
4 de ellos, siendo de corta evolución en 3 y de curso más prolongado en uno. En 7 pacientes no hubo secuelas aparentes en el momento del alta; en uno quedó una hemiplegia $y$ en otro una fina membrana subdural correspondiente al que desarrolló derrarne subdural prolongado.

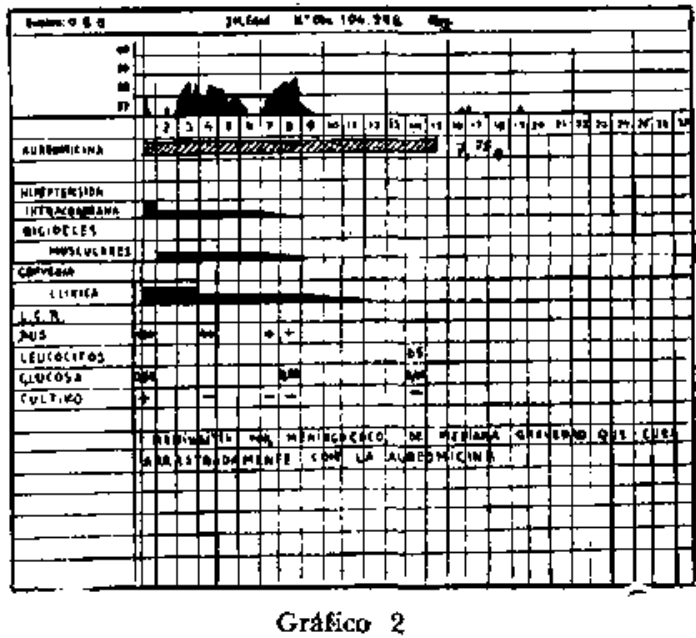

En el cuadro 3 se analizan los enfermos del Grupo II, en quienes fracasó el tratamiento exclusivo a base de aureomicina. Como en el Grupo I, la gravedad de estos pacientes fué variable de un caso a otro. Las causas de la interrupción del medicamento fueron: la intolerancia manifestada por vómitos intensos (caso 13); la ineficacia total del tratamiento, traducida por la agravación del cuadro clínico y acción casi nula sobre las alteraciones del LCR (casos 6 y 8), y una eficacia sólo parcial de la droga (casos 7, 9, $14 \mathrm{y}$ 16) sin obtenerse una regresion total del cuadro clínico o de las alteraciones del ICR. En los casos 7 y 9 , que recibieron tratamiento por un lapso de 23 y 14 dias, respectivamente, se produjo la reaparición del germen en uno de ellos durante la administración de la droga y en el otro después de interrumpida. En Ios otros dos casos (14 y 16) cuyo tratamiento duró 21 y 5 días respectivamente, se produjo durante él una reagudización clínica y aumento de las alteraciones del LCR después de una mejoría aparente. En los gráficos 3 y 4 puede observarse la evolución seguida por dos enfermos en los cuales hubo que interrumpir la droga.

Caso 6. (gráfico 3). Esta observación coriesponde a un lactante de 6 meses de edad, 
GUADRO N. 3

Algunos caracteres de los enfermos de meningitls aguda purulenta tratados sin éxito con aureomicina

\begin{tabular}{|c|c|c|c|c|c|}
\hline N. ${ }^{\circ}$ & Etiologta & Gravedad & $\begin{array}{l}\text { Durac. } \\
\text { del } \\
\text { tratam. } \\
\text { (días) }\end{array}$ & $\begin{array}{c}\text { Causa de interrupción } \\
\text { del tratamiento }\end{array}$ & Secuelas \\
\hline $\begin{array}{l}6 \\
7\end{array}$ & $\begin{array}{l}\text { D. pneumoniae } \\
\text { D. pneumoniae } \\
\text { y } \mathrm{H} \text {. influenzae }\end{array}$ & $\begin{array}{l}++ \\
+++\end{array}$ & $\begin{array}{r}5 \\
23\end{array}$ & $\begin{array}{l}\text { Ineficacia total } \\
\text { Eficacia parcial }\end{array}$ & $\stackrel{(-)}{\text { Hidrocefalia }}$ \\
\hline $\begin{array}{r}8 \\
9 \\
13 \\
14 \\
16\end{array}$ & $\begin{array}{l}\text { H. influenzae } \\
\text { H. influenzae } \\
\text { Sin germen } \\
\text { Sin germen } \\
\text { Sin germen }\end{array}$ & $\begin{array}{l}+++ \\
+++ \\
++ \\
++ \\
++\end{array}$ & $\begin{array}{l}2,5 \\
14 \\
6 \\
21 \\
5\end{array}$ & $\begin{array}{l}\text { Ineficacia total } \\
\text { Eficacia parcial } \\
\text { Intolerancia } \\
\text { Eficacia parcia] } \\
\text { Eficacia parcial }\end{array}$ & $\begin{array}{l}\text { Hemipelgia derecha } \\
\text { Hemiplegia izquierda } \\
\text { Hidrocefalia } \\
\text { Retardo p sicomotor }\end{array}$ \\
\hline
\end{tabular}

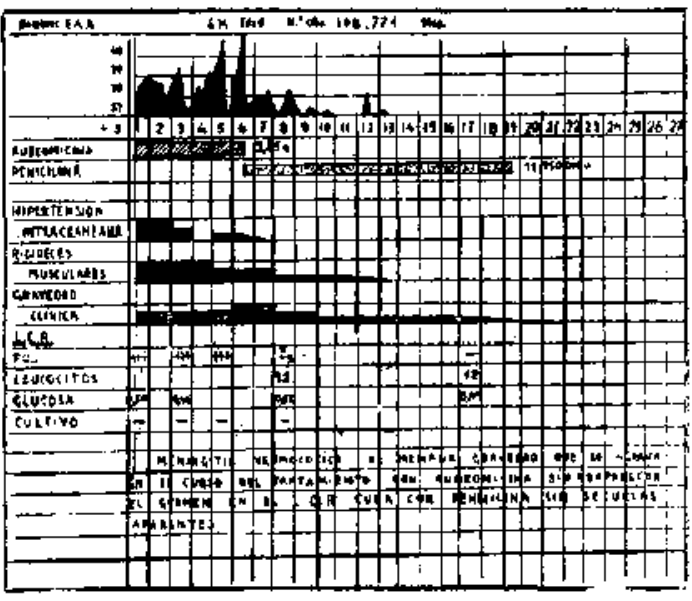

Gráfico 3

que sufre una meningitis neumocócica con compromiso general de mediana intensidad en quien el efecto de la aureomicina sólo se tradujo en la esterilización del LCR y atenuación discreta del síndrome meaíngeo. La fiebre se hizo cada vez más alta y el compromiso del estado general y psíquico se intensificó. El cuadro infeccioso cedió rápidamente bajo la acción de la penicilina sódica curando sin dejar secuelas aparentes.

Caso 9 (gráfíco 4). En este gráfico puede verse la evolución seguida por un enfermo de 20 meses de edad que ingresó muy grave con una neumonía en evolución y la meningitis por $H$. influenzae complicada con una hemiplegia izquierda. Bajo la acción de la aureomicina oral se obtuvo la desaparición de la fiebre en 2 días; la mejoría del LCR hasta una fórmula de recuperación en forma bastante rápida y la curación del proceso neumónico. Sin embargo, el niño continuó con cierto grado de compromiso del estado general y rigidez de la nuca. $\mathrm{A}$ los $\mathbf{1 6}$ días se interrumpió la administración de la aureomicina y entonces reapareció la fiebre alta y el germen en el LCR. Se trató en esta ocasión con estreptomicina y sulfadiazina obteniéndose la curación en forma algo arrastrada y quedando como secuela la hemiplegía ya descrita.

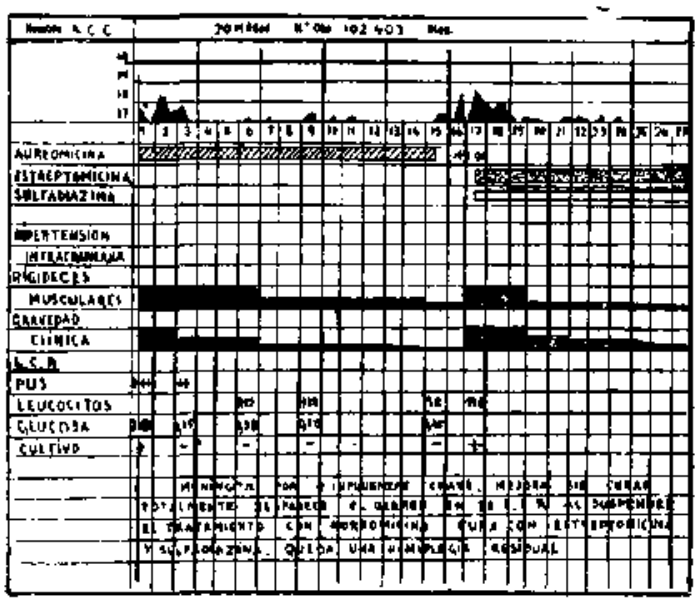

\section{Gráfico 4}

Es dificil interpretar las causas precisas del fracaso del tratamiento en una parte de nuestro material. Es importante destacar que en tres de nuestros pacientes en quienes hubo que interrumpir el tratamiento, el germen etiológico fuera el $\mathbf{H}$. influenzae, siendo que en la experiencia extranjera (2-4-6) se ha demostrado la eficacia de la aureomicina en el tratamiento de la meningitis causada por dicho microorganismo. Es posible tal vez que no se obtengan siempre niveles útiles en el LCR después de la administra- 
ción oral de la aureomicina. La presencia de vómitos esporádicos $\mathrm{y}$ de diarrea discreta aparecida aproximadamente en la mitad de rivestra casuística haría más importante esta situación a pesar que en nuestros enfermos estas manifestaciones se produjeron en ambos ǵrupos con más o menos igual intensidad. Es de interés recordar al respecto que en el espacio subaracnoídeo la fagocitosis es poco intensa (8) por lo cual serían necesarios niveles de antibióticos más altos. Puede sugerirse que la vía endovenosa que produce niveles más elevados constituya una norma terapéutica aconsejable, por lo menos, en los primeros días de la enfermedad.

\section{Resumen}

Se tratan con aureomicina oral 16 enfermos de meningitis purulenta de diversa etiología. Se obtiene la curación con aureomicina exclusiva en 9 casos, 2 de los cuales quedan con secuelas (hemiplejia, membrana subdural). En 7 casos es necesario interrumpir la administración de la droga por intolerancia en 1 caso; por ineficacia total en 2 , y por una eficacia sólo parcial en 4. Se analizan los resultados y se discuten las posibles causas del fracaso de la aureomicina en una parte de los enfermos.

\section{Summary}

16 patients with purulent meningitis of different ethiology are treated with oral aureomycin. A cure with aureomycin exclusively, is obtained in 9 cases, 2 of which remain with sequelas (hemiplegia, subdural membrane). In 7 cases it was necessary to interrupt the administration of the drug due to intolerance in 1 case; complete uselessness in 2; and partial results in 4. The results are analyzed and the possible causes of failure of the aureomycin in some patients is discussed.

\section{AIBLIOGRAFIA}

1. AGUILO, C. y MENEGHELLO, J-Tratamiento de las meningitis agudas purulentas del lactante con exclusión de la vía intrarraquídea. Rev. Chil. de Pediatrfa 21: 486, 1950.

2. DRAKE, M. E., BRADLEY, J, E., IMBURG, J., Mc, CRUMB, F. R. Jr. Y WOODWARD, T. EAureomycin in the Treatment of Influenzal Meningitis, J. A. M. A. 142: 468,1950 .

3. HARVEY, S, C., MIRICK, G, S. y SCHAUB, J. G.-Clinical Experience with Aureomycin. J. Clin. Invest. 28: 987, 1949.

4. JOSLIN, B. S. Y HOWARD, A.-Haemophylus Influcnzae Pyartrosisi and Meningitis Treated with Aureomycin. J. Pediat. 38: 375, 1951.

5. LEPPER, M. H. y DOWLING, H. F.-Treatment of neumococcic Meningitis with Penicillin Com. pared with Penicillin plus Aureomycin, Arch. Int. Med. 88: 489, 1951.

6. MC. GOVERN, J. J.4 MC. GOVERN, P. J., ROSS, S., RICE, C. E. y BURKE, F. G.-Auteomycin in the Treatmen of Influenzae Meningitis, A Comparison of Therapy in 54 Consecutive Cases overca 3 Years Pcriod. Clin. Proc. 8: 69, 1952.

7. MENEGHELLO, J. y AGLILO, C-Meningitis aguda no meningocócica en el niño menor de 3 afnos. Experiencia en 161 casos tratados con antibiólicos. Rev. Chi]. de Pediatría 22: 496, 1951.

8. SMITH. M. R. PERRY, W. D., BERRY, J. $W$. y WOOD, W. B. Jr.-Surface Phagocytosis in Vivo. J. Inmunol. 67: 71, 1951 .

9. SCHOENBACH, E. B.-The Clinical Use of Aurenmycin. Citado por Lepper y col. (5).

10. WHITLOCK, C. M. Jr., HUNT, A. D. Jr, y TASHMAN, 5.-Studies on the Administration, Absortion, Distribution and Excretion of Aureomycyn in Children. With Observations Concerving Tolerance, Dosage Shedules and Certain Therapeutics Indications. Pediatrics 6: 827, 1950. 